\title{
328 BATF3 DENDRITIC CELLS AND 4-1BB/4-1BB LIGAND AXIS ARE REQUIRED AT THE EFFECTOR PHASE WITHIN THE TUMOR MICROENVIRONMENT FOR ANTI-PD-L1 EFFICACY
}

${ }^{1}$ Andrea Ziblat*, ${ }^{2}$ Brendan Horton, ${ }^{1}$ Emily Higgs, ${ }^{1}$ Ken Hatogai, ${ }^{1}$ Thomas Gajewski. ${ }^{1}$ University of Chicago, Chicago, IL, USA; ${ }^{2}$ MIT, Cambridge, MA, USA

Background PD-1/PD-L1 blockade has shown clinical benefit across many cancer types. However, a large fraction of patients are resistant to immune checkpoint blockade therapy and others eventually relapse. Understanding the mechanisms involved in $\alpha$ PD1/PD-L1 immunotherapy efficacy may enable new strategies for improving clinical outcomes. Given that Batf3-lineage dendritic cells (DCs) are needed for spontaneous $\mathrm{T}$ cell priming in the tumor-draining lymph node and for recruitment of effector $\mathrm{CD} 8+\mathrm{T}$ cells to the tumor, in the current work we examined whether Batf3+ DCs are also required during the effector phase of the anti-tumor immune response at the time of anti-PD-L1 administration for therapeutic efficacy.

Methods We utilized the B16-SIY melanoma model, CD11cDTR-GFP, and CD11c-DTR-GFP/Batf3 KO bone marrow chimeras to study the role Batf3 + DCs play during anti-PD-L1 immunotherapy. To focus on the effector phase of the immune response, we depleted $\mathrm{CD} 11 \mathrm{c}+$ cells with diphtheria toxin from day seven of tumor injection while simultaneously blocking new $\mathrm{T}$ cell entry with FTY720. As flow cytometry revealed high 4-1BBL expression on intratumoral Batf3-DCs, 4-1BB KO mice and anti-4-1BBL blocking antibodies were used. Tumor growth and phenotypic analysis of the tumor infiltrate were evaluated.

Results Strikingly, we observed that CD11c+ cells, and specifically Batf3 + DCs, were required in the tumor prior to $\alpha \mathrm{PD}$ L1 treatment for immunotherapy efficacy. The normal intratumoral expansion of antigen (Ag)-specific CD8 + tumor-infiltrating $\mathrm{T}$ cells (TILs) and increased ratio between Ag-specific CD8 + TILs and regulatory $\mathrm{T}$ cells following anti-PD-L1 therapy was eliminated with Batf3 + DC depletion. Batf3+ DCs expressed high levels of 4-1BBL, and increased expression of $4-1 \mathrm{BB}$ on antigen-specific CD8 + TILs upon $\alpha \mathrm{PD}-\mathrm{L} 1$ treatment required Batf3 + DCs. Mechanistic experiments confirmed a requirement for $4-1 \mathrm{BB}$ expression on immune cells for $\alpha \mathrm{PD}$ L1 efficacy, and blocking antibodies against 4-1BBL eliminated anti-PD-L1 efficacy as well. Using appropriate bone marrow chimeras, agonistic 4-1BB antibodies were sufficient to bypass the need for CD11c+ DCs at the effector phase for tumor control. In human melanoma samples, co-localization of Batf3 + DCs and CD8 $+\mathrm{T}$ cells was observed in $\mathrm{T}$ cell-inflamed tumors, which correlated with anti-PD-1 efficacy in metastatic melanoma.

Conclusions Our results indicate that Batf3 + DCs are necessary during the effector phase of the anti-tumor immune response for anti-PD-L1 efficacy to occur, at least in part through 4-1BB/4-1BBL-mediated reinvigoration of Ag-specific CD8 + TILs.

Ethics Approval The study obtained ethics approval, IRB protocol 15-0837.

http://dx.doi.org/10.1136/jitc-2021-SITC2021.328 